\title{
O efeito das UPAs na taxa de internações por condições sensíveis à atenção primária
}

\author{
Rodrigo de Vasconcellos Viana Medeiros ${ }^{1}$ \\ João Gabriel Alves da Costa ${ }^{2}$ \\ Leonardo Chaves Borges Cardoso ${ }^{3}$
}

\section{Resumo}

Este artigo tem como objetivo avaliar o efeito das Unidades de Pronto Atendimento (UPA) sobre as internações por condições sensíveis à atenção primária (ICSAP) nos municípios do estado do Rio de Janeiro. Para alcançar o objetivo proposto, a estratégia empírica utilizada consistiu em elaborar um modelo de diferenças em diferenças (DD) organizado ao nível municipal entre os anos 2000 a 2017, identificando as ICSAP a partir do município de residência do indivíduo. Foi constatado que as UPAs têm um efeito negativo e significativo na taxa de ICSAP, caracterizando essa estrutura de saúde como um instrumento relevante capaz de reduzir a superlotação em emergências hospitalares. Em média, os municípios com UPA instalada reduziram as ICSAP em cerca de 21 unidades por 100 mil habitantes ao longo do período em que houve as inaugurações das UPAs. Tais resultados fornecem evidências da importância das UPAs no atual sistema brasileiro de atenção às urgências e emergências.

\section{Palavras-chave}

Unidades de Pronto Atendimento. Internações hospitalares. Diferenças em Diferenças.

\begin{abstract}
This article aims to evaluate the effect of Emergency Care Units (UPA) on hospitalizations for conditions sensitive to primary care (ICSAP) in the municipalities of the state of Rio de Janeiro.
\end{abstract}

- O presente trabalho foi realizado com o apoio da Coordenação de Aperfeiçoamento de Pessoal de Nível Superior - Brasil (CAPES) - Código de Financiamento 001.

1 Doutorando em Economia Aplicada - Universidade Federal de Viçosa (UFV) - Departamento de Economia Rural - End.: Avenida Purdue, s/n, Edifício Edson Potsch Magalhães - Campus Universitário Centro - CEP: 36570-900, Viçosa-MG-Brasil-E-mail: rodrigodevasconcellos.medeiros@gmail.com ORCID: https://orcid.org/0000-0002-2675-923X.

2 Mestre em Economia Aplicada - Universidade Federal de Viçosa (UFV) - Departamento de Economia Rural - End.: Avenida Purdue, s/n, Edifício Edson Potsch Magalhães - Campus Universitário Centro - CEP: 36570-900 - Viçosa - MG - Brasil - E-mail: gabrieldorathe@gmail.com ORCID: https://orcid.org/0000-0002-0101-9200.

3 Professor Adjunto - Universidade Federal de Viçosa (UFV) - Departamento de Economia Rural End.: Avenida Purdue, s/n, Edifício Edson Potsch Magalhães - Campus Universitário - Centro CEP: 36570-900 - Viçosa - MG - Brasil - E-mail: leonardocardoso005@gmail.com ORCID: https://orcid.org/0000-0002-5757-107X. Recebido: 17/01/2021. Aceito: 06/04/2021. Editor Responsável: Marcos Yamada Nakaguma

(c) (i) (\$) Esta obra está licenciada com uma Licença Creative Commons Atribuição-Não Comercial 4.0 Internacional. 
To achieve the proposed objective, the empirical strategy used consisted of elaborating a model of differences in differences (DD) organized at the municipal level between the years 2000 to 2017, identifying the ICSAP from the individual's municipality of residence. It was found that UPAs have a negative and significant effect on the rate of ACSC, characterizing this health structure as a relevant instrument capable of reducing overcrowding in hospital emergencies. On average, the municipalities with installed UPA reduced the ICSAP by approximately 21 units per 100 thousand inhabitants during the period in which the UPAs was inaugurated. These results provide evidence of the importance of UPAs in the current Brazilian system of attention to urgencies and emergencies.

\section{Keywords}

Emergency Care Units. Hospitalizations. Diff-in-Diff.

\section{JEL Classification}

112. I18. C23.

\section{Introdução}

O início do ano 2020 foi marcado pela grande preocupação dos governos de todo o mundo com a possibilidade de superlotação de seus hospitais devido ao avanço da pandemia causada pelo novo coronavírus. No Brasil, segundo Silva et al. (2012) a superlotação nas portas das emergências hospitalares é um fenômeno bem conhecido dos gestores e usuários do sistema público de saúde (SUS). Com seu impacto negativo para os pacientes, essa superlotação constitui um motivo de crítica ao modelo assistencial à saúde no Brasil. As médias e grandes regiões metropolitanas têm optado pela organização de uma rede assistencial às urgências através da atenção básica seguindo as Políticas Nacionais de Atenção às Urgências (PNAU) e de Humanização (PNH).

Embora não exista um sistema de saúde ideal, existem modelos como em Portugal e Espanha, que são semelhantes ao sistema do SUS, porém encontram-se mais avançados em termos de atendimento. Nestes sistemas, a atenção básica de saúde tem ampla cobertura e encontra-se bem estruturada, facilitando a interação com os demais níveis de especialização (Fiocruz, 2015). O foco do sistema nestes países está na alta resolutividade de problemas de saúde que são considerados básicos, evitando que estes se tornem internações. O Brasil, segundo o Ministério da Saúde (2014), também vem tentando adotar essa estratégia com a ampliação da atenção 
básica. Como o objetivo é evitar que problemas mais simples virem internações e sabendo que a demanda potencial por um leito de internação é enorme, não faz sentido avaliar o sucesso do programa pela redução no tamanho de filas, ou taxas de ocupação dos leitos. Daí surge a importância de se investigar o perfil das internações hospitalares, na tentativa de compreender mais profundamente a demanda por esse tipo de serviço.

Nas últimas décadas, ocorreu um aumento constante na utilização pelos serviços hospitalares de emergência, onde mais da metade dessa procura poderia ser resolvida com procedimentos ambulatoriais na atenção básica de saúde. A baixa resolutividade na atenção primária provoca em muitos hospitais a ocupação de $100 \%$ dos leitos de internação, ocasionando um excesso de demanda nesses estabelecimentos de saúde, e sobrecarregando profissionais e equipamentos que poderiam ser utilizados em processos clínicos mais complexos (O’dwyer, Oliveira e Seta, 2009).

Esse excesso de demanda, segundo Iunes (1995), é complicado de ser administrado uma vez que, do ponto de vista do indivíduo, a procura por serviços de saúde é frequentemente irregular ou imprevisível. Além disso, a demanda por saúde ocorre em uma situação anormal para o indivíduo (a doença) o que pode comprometer sua racionalidade de decisão. Outro fator destacado pelo autor se refere a características não monetárias que existem na demanda por saúde, como o tempo gasto para consumir tais serviços. Se tratando de uma necessidade imediata, locais em que não exista um atendimento de urgência para a população podem não só afetar negativamente as condições de saúde em cada indivíduo, como também provocar distorções na oferta e demanda desses serviços.

Neste contexto, surgem as Unidades de Pronto Atendimentos (UPAs) que, segundo a Secretaria Estadual de Saúde/RJ (SES/RJ, 2010), são uma organização pública, com sistema complexo, que tem como atividade-fim o atendimento ao usuário que necessita de imediatismo nos cuidados de sua saúde. As UPAs têm como objetivo atender às regiões desprovidas desta modalidade de pronto-atendimento e exames correlatos, reduzindo a espera para realização dos mesmos, evitando o deslocamento desnecessário e excessivo dos pacientes, melhorando o atendimento assistencial e reduzindo a sobrecarga das unidades hospitalares do Estado. 
Esse alívio da sobrecarga nos hospitais se daria a partir dos atendimentos de complexidade intermediária realizados pela UPA, ou seja, as urgências ligadas ao infarto, derrame, fraturas, infecções respiratórias, pediatria, cólicas renais e pressão arterial seriam concentradas nessas unidades, o que reduziria as internações por condições sensíveis à atenção primária (ICSAP). Entretanto, para o seu adequado funcionamento, essa estrutura precisa de apoio técnico, logístico e administrativo compreendido por uma interligação sólida entre a atenção básica e o SAMU, Serviço de Atendimento Móvel de Urgência (Ministério da Saúde, 2011; SES/RJ, 2010).

Enquanto muitos estudos como em Boing et al. (2012), Moura et al. (2010), Zarlotti et al. (2017), entre outros, enfatizam a importância de se ampliar os cuidados primários na atenção básica, com mais Unidades Básicas de Saúde (UBS) para atender a população tendo em vista seus potenciais em reduzir as ICSAP. Mesmo assim, pouco se sabe da relevância das UPAs nesse processo. Do ponto de vista institucional, sua importância é indiscutível, pois traz uma nova porta de entrada ao sistema público de saúde brasileiro. Porém, na literatura empírica, não há estudos sobre o impacto dessa estrutura de saúde na demanda por internações em hospitais. As exceções são Pinto, Stocker e Lima (2019) e Lima, Nichiata e Bonfim (2019), que realizam análises sobre o desempenho das UPAs. Nestes fica evidenciado a dificuldade das UPAs em absorver todos os atendimentos por ICSAP.

Diante desse contexto, o presente trabalho tem como objetivo avaliar o impacto das Unidades de Pronto Atendimento (UPAs) na demanda por internações sensíveis à atenção primária nos municípios fluminenses através da aplicação do método de diferenças em diferenças. A demanda por serviços de saúde sofre um impacto negativo devido à sobrecarga na prestação de tais serviços. Dessa forma, indivíduos podem desistir de buscar serviços de saúde por conta de longas filas de espera para exames, consultas médicas, entre outros serviços. Do ponto de vista econométrico, isto pode gerar problemas, pois, caso a qualidade dos serviços melhore, novos consumidores adentrariam ao mercado, dificultando a identificação da efetividade dos atendimentos nas UPAs.

De maneira semelhante, o efeito das UPAs poderia ser superestimado caso as internações fossem mensuradas pelo local de ocorrência, pois a população de municípios que não são atendidos por essa estrutura seria capaz de recorrer a municípios vizinhos que tivessem tais instalações. Corrigir 
esse tipo de viés se tornaria complexo, dado que seria necessário definir empiricamente uma distância entre as cidades para o efeito potencial das UPAs.

Este trabalho contribui ao avançar em ambos os problemas. Primeiro, ao invés de olhar para o efeito das UPAs em variáveis ligadas ao número total de atendidos, verificará o impacto dessas unidades no perfil dos atendimentos. Segundo, a internação dos indivíduos foi contabilizada a partir do seu local de residência. Desse modo, evita-se que a análise se torne sensível a pessoas que saíram de seu município para serem atendidas em outros locais com UPA implantada (efeito migração). Conhecendo os impactos dessa estrutura pré-hospitalar nas internações, é possível que os gestores municipais e estaduais aloquem estrategicamente seus recursos humanos, financeiros e físicos em ações voltadas para políticas de atenção às urgências de saúde.

Além desta introdução, o artigo contém mais cinco seções. Na seção seguinte é realizada uma revisão de literatura sobre o surgimento das UPAs e suas relações com a atenção primária de saúde. A terceira seção apresenta os motivos que os indivíduos demandam serviços de saúde. Já a quarta seção destina-se em explicar a estratégia empírica utilizada no estudo. $\mathrm{Na}$ quinta seção são apresentados resultados obtidos. Por fim, a última seção realiza algumas considerações finais e indica direções para futuras pesquisas.

\section{As UPAs e sua relação com a atenção básica e internações hospitalares}

As UPAs surgiram em 2007 no município do Rio de Janeiro como iniciativa do governo do Estado, pretendendo responder ao contexto de crise na saúde do município que teve seu ápice em 2005. A partir daí, as UPAs foram implantadas de forma bastante acelerada, primeiro na capital e, posteriormente, no restante do estado. Somente no final de 2008 houve a primeira portaria regulando as UPAs na esfera federal (Konder e O'dwyer, 2016).

Segundo O'Dwyer et al. (2017), as implantações das UPAs previam uma integração estratégica com a rede de atenção às urgências. Isso significa que 
seria necessária uma coexistência com o SAMU e uma ampliação obrigatória da cobertura do programa de Estratégia de Saúde da Família (ESF) para que as demandas atendidas nas UPAs tivessem alta resolutividade e não se transformassem nos antigos prontos socorros, notavelmente produtores de consultas de emergência pouco resolutivas.

A UPA é considerada um componente pré-hospitalar fixo, de complexidade intermediária o que a situa entre a atenção primária e o sistema hospitalar. Por esse motivo, suas competências são: a) prestar atendimento qualificado e resolutivo em quadros clínicos agudos ou crônicos; b) realizar consulta médica em regime de pronto atendimento nos casos de menor gravidade; c) acolher sempre as demandas de atendimento; d) prestar primeiro atendimento aos quadros cirúrgicos e de trauma e manter em observação clínica por até 24 h para elucidação diagnóstica ou estabilização; e) encaminhar para internações em hospitais pacientes com quadros não resolvidos após 24h (Konder, 2013).

Desse modo, possíveis urgências de complexidade intermediária e que não foram sanadas na atenção básica são passíveis de resolução em sua estrutura, evitando que se tornem internações hospitalares. Segundo Moura et al. (2010), as principais causas de internações por condições sensíveis à atenção primária (ICSAP) entre os mais jovens (até 20 anos) no Brasil como um todo são decorrentes de gastroenterites, doenças respiratórias crônicas (asma) e infecções pulmonares bacterianas. Todas essas enfermidades são tratáveis e solucionáveis em uma UPA, tendo em vista que esta estrutura contém aparelhagem e profissionais para iniciar os devidos tratamentos em estágios não avançados dessas doenças. Ainda segundo Moura et al. (2010), entre os anos 1999 e 2006, período anterior as UPAs, as regiões do Brasil apresentaram, no agregado, uma tendência irregular de pequenas quedas e ligeiros aumentos nas taxas (ICSAP) em jovens com até 20 anos. Doenças como pneumonia bacteriana e gastroenterite sofreram picos de crescimento acentuado, caindo suavemente ao longo dos anos.

Em um estudo considerando a população de todas as idades, Boing et al. (2012) mostraram através de uma análise de séries temporais que, entre 1998 e 2009, houve redução na média anual de ICSAP nos estados brasileiros tanto em homens como em mulheres. A principal redução, em ambos os sexos, foi encontrada para doenças ulcerativas gastrointestinais, seguida das doenças respiratórias das vias inferiores. Além disso, foi possível observar que em algumas doenças (asma, úlcera gastrointestinal e infecções 
da pele e tecido subcutâneo) a tendência de queda se acentuou nos períodos 2008 e 2009, período em que ocorreram as primeiras implantações das UPAs. Ainda que os autores não tenham tratado dessas unidades em seu estudo, essas quedas podem ser indícios dos primeiros impactos das UPAs nas internações hospitalares sobretudo se observado que o estado do Rio de Janeiro apresentou uma das maiores variações médias de ICSAP.

Figueiredo et al. (2012) e Lima, Nichiata e Bonfim (2019) relatam que as UPAs resolvem, em média, 85\% dos casos clínicos de pacientes dentro de suas unidades e pelo menos a metade desses casos estão relacionadas à atenção primária. Isso revela a insuficiência das Unidades Básicas de Saúde (UBS) em atender a demanda da população e a importância da atenção secundária no sistema de saúde brasileiro. Ainda segundo os autores, na ausência dessas unidades muitos desses casos evoluíram para situações mais graves, levando o indivíduo a procurar hospitais públicos. Logo, a relevância dessas unidades está em evitar agravo dessas enfermidades e aliviar a demanda nos hospitais públicos de cada localidade.

Especificamente para o estado do Rio de Janeiro, a literatura carece de informações de como as UPAs podem contribuir para que as ICSAP tenham suas taxas reduzidas ou ainda como essas unidades podem reduzir os custos com internações evitáveis. Segundo o Departamento de Informática do SUS (Datasus, 2018), o custo médio com ICSAP em hospitais nos municípios fluminenses é $\mathrm{R} \$ 1.017,77$. Considerando que a maioria dos atendimentos nas UPAs ocorre em função dessas doenças, o funcionamento adequado dessas unidades pode representar uma vantajosa economia para os cofres públicos.

Alguns trabalhos mais recentes sobre ICSAP como Silva e Gonçalves (2019), Santos et al. (2018) e Zarlotti et al. (2017) caracterizam com maiores detalhes o comportamento da ICSAP. Todos esses estudos foram realizados em municípios fluminenses e apresentaram características semelhantes quanto à tendência de redução ${ }^{1}$ das ICSAP em hospitais da rede pública de saúde. A partir de 2011, ano em que as UPAs consolidaram sua expansão pelo interior do estado, a taxa por ICSAP começou a apresentar quedas maiores. Foi também durante esse período que o programa Estratégia Saúde da Família (ESF) obteve maior expansão, onde foram observados um crescimento na cobertura de famílias com atendimentos

1 Algumas doenças como insuficiência cardíaca e infecções da pele e tecidos subcutâneos tiveram um ligeiro aumento no estudo realizado por Silva e Gonçalves (2019) em Miguel Pereira. 
em Unidades Básicas de Saúde (UBS). Logo, fica evidente que a ampliação de cobertura das UBS aliada com os serviços de urgências intermediárias das UPAs podem fornecer um importante apoio para os hospitais da rede pública na mitigação da superlotação dos leitos hospitalares.

\section{Fatores que afetam a demanda por saúde: uma breve revisão}

A partir da segunda metade do século passado, os estudos sobre a economia da saúde se atentaram às contribuições da teoria da demanda para explicar a busca por cuidados médicos, assim como identificar possíveis falhas de mercado que eventualmente surgissem. Nesse sentido, vários estudos emergiram propondo que as análises não incluíssem apenas variáveis relacionadas ao mercado de assistência médica (como o preço e a oferta dos serviços), mas também incorporar o próprio status de saúde, as características dos indivíduos e o ambiente no qual estão inseridos.

Com o surgimento de diversos debates nessa área, a referência seminal para o tema foi proposta por Grossman (1972). Em seu modelo inspirado na teoria do capital humano de Gary Becker, o autor introduziu o conceito de saúde como um bem durável de capital que é herdado, mas que se deprecia ao longo do tempo. Sendo assim, é necessário que o indivíduo realize investimentos em saúde. Este, por sua vez, é tido como uma atividade onde os cuidados médicos são combinados com outros insumos para a "produção" de um novo status de saúde que seja, pelo menos, suficiente para repor a sua deterioração natural.

Entre os resultados encontrados por Grossman (1972), destaca-se que a demanda por saúde está relacionada positivamente com os cuidados médicos e com a escolaridade dos indivíduos, ou seja, quão mais saudáveis os indivíduos estiverem, maior será a sua busca por serviços médicos. Por outro lado, trabalhos como Wagstaff (1993) e Galama e Kapteyn (2011) apontam que fatores ambientais e as condições socioeconômicas podem ser mais decisivos para os indivíduos. Segundo Galama e Kapteyn (2011), a relação entre status de saúde e serviços médicos é negativa e fatores como o tempo de espera pelo atendimento médico e o custo do tratamento pós intervenção médica são importantes para explicar a demanda por serviços de saúde. 
No contexto brasileiro, Castro, Travassos e Carvalho (2005) analisaram a demanda por serviços hospitalares nas regiões metropolitanas de cada estado. A pesquisa mostrou que o principal fator que leva adultos e/ou crianças a uma hospitalização é a necessidade em melhorar seu status de saúde. Além disso, os autores também identificaram que os indivíduos com menor nível de renda possuem mais chances de ficarem internados. Outro resultado relevante está ligado ao papel que o atendimento ambulatorial possui nesse contexto. Segundo os autores, regiões em que a cobertura do atendimento ambulatorial era maior foi encontrada uma associação inversa com as admissões hospitalares.

Para Politi (2014), a desigualdade na utilização dos serviços de saúde é um dos principais desafios do sistema de saúde público no Brasil. Em seu estudo, o autor apontou que procedimentos de alta complexidade, como cirurgias, e de média complexidade (como consultas especializadas) são os serviços em que existem uma grande concentração em favor dos mais ricos. Em contraposição, a melhoria de fatores ambientais nos últimos anos, como melhores condições de acesso à água potável e tratamento de esgoto, assim como os níveis de escolaridade e expansão das políticas públicas de saúde como os Postos de Saúde da Família (PSF), contribuíram, mesmo que modestamente, para que os mais pobres pudessem ter suas demandas de saúde básica minimamente atendidas.

Brito, Andrade e Perpétuo (2006) também contribuem para o tema ao analisar os determinantes da procura individual por cuidados médicos. Utilizando como controle características socioeconômicas e demográficas, os autores identificaram que o processo decisório passa por fases distintas. $\mathrm{Na}$ primeira fase, que envolve a procura pelos serviços de saúde, a idade e o sexo foram importantes para identificar que mulheres e pessoas mais idosas buscam mais pelos serviços médicos. Na segunda fase, que envolve decisões em conjunto com o médico sobre o tipo de tratamento, a proxy que media o status de saúde individual se mostrou relevante quanto a decisão de hospitalização, ou seja, os indivíduos que gastaram mais com serviços de saúde ou que já haviam sido internados em períodos anteriores tinham maiores chances de internação.

Como foi visto, existe uma série de variáveis que podem potencializar a depreciação ou a melhoria do estado de saúde da população e, nesse sentido, é necessário que essas características sejam controladas adequadamente para que os efeitos das políticas públicas em saúde, como no caso da UPA, sejam devidamente identificadas. 


\section{Estratégia Empírica}

\subsection{Dados e variáveis de estudo}

Para avaliar o impacto das UPAs sobre a demanda por ICSAP inicialmente foi feito um levantamento com as informações sobre a quantidade desses estabelecimentos nos 92 municípios do Rio de Janeiro. A fonte de dados é pública e obtida através do Cadastro Nacional de Estabelecimento de Saúde (CNES) no site do Datasus ${ }^{2}$. Desde a primeira unidade instalada, em 2007, até a última atualização disponível do CNES (2019) o estado do Rio de Janeiro possuía 114 UPAs em funcionamento espalhadas por 41 municípios fluminenses. A data de inauguração de cada unidade foi obtida através da imprensa local por meio da divulgação de notícias sobre inauguração das UPAs.

A variável explicada no estudo também foi obtida no Datasus e se refere ao número de ICSAP aprovadas no sistema público de saúde. Isso significa que hospitais públicos e privados foram incluídos, haja vista que muitas entidades particulares possuem convênio para atender demandas provenientes do SUS. É importante destacar que o Datasus fornece informações sobre internações tanto por local de residência como por local de ocorrência. Como estratégia, optou-se em obter as ICSAP por local de residência, pois dessa forma evita-se que sejam quantificadas internações de indivíduos que tenham saído de seu município em busca de serviços médicos em outras cidades onde existem UPAs instaladas. Desse modo, é possível isolar qualquer efeito de migração que venha a existir.

Outras variáveis foram incluídas no estudo como tentativa de controlar as demais fontes de variação que interferem na deterioração da saúde dos indivíduos conforme os estudos comentados anteriormente. Portanto, foram inseridas variáveis relacionadas ao saneamento básico, renda e escolaridade, condições socioeconômicas as quais os indivíduos estão sujeitos continuamente. $\mathrm{O}$ quadro 1 resume as informações e as fontes de todas as variáveis incluídas no estudo.

2 Disponível em: < http://cnes2.datasus.gov.br/Mod_Ind_Unidade.asp > . Acesso em 15 de abr. 2020. 
Quadro 1 - Variáveis utilizadas no estudo

\begin{tabular}{|c|l|c|c|}
\hline Variável & \multicolumn{1}{|c|}{ Definição } & Fonte & Sinal esperado \\
\hline ICSAP & $\begin{array}{l}\text { Variável dependente que representa a taxa de ICSAP por } \\
100 \text { mil habitantes e por local de residência de acordo com } \\
\text { lista de Classificação Internacional de Doenças }{ }^{1} \text { (CID-10) }\end{array}$ & Datasus & \\
\hline Ag & $\begin{array}{l}\text { Variável de controle que representa o índice (percentual) } \\
\text { de atendimento urbano de água no município. É calculado } \\
\text { pela relação entre a população total atendida com água e } \\
\text { a população residente total do município. }\end{array}$ & $\begin{array}{c}\text { Sistema Nacional de } \\
\text { Informaçães sobre } \\
\text { Saneamento (SNIS) }\end{array}$ & $(-)$ \\
\hline Esg & $\begin{array}{l}\text { Variável de controle que representa o índice (percentual) } \\
\text { de atendimento total de esgoto no município. É calculado } \\
\text { pela relação entre a população total atendida com esgota- } \\
\text { mento sanitário e a população residente total do município. }\end{array}$ & SNIS & $(-)$ \\
\hline PIB & $\begin{array}{l}\text { Variável de controle que indica o PIB per capita a preçoS } \\
\text { constantes do ano 2000 como medida de riqueza muni- } \\
\text { cipal. }\end{array}$ & IBGE \\
\hline NMAT & $\begin{array}{l}\text { Variável de controle que representa a taxa por mil habi- } \\
\text { tantes de alunos matriculados cursando e concluintes em } \\
\text { alguma instituição de ensino superior. }\end{array}$ & $\begin{array}{c}\text { Instituto Nacional de } \\
\text { Estudos e Pesquisas } \\
\text { Educacionais }\end{array}$ & $(-)$ \\
\hline ESF & $\begin{array}{l}\text { Variável de controle que representa o percentual que as } \\
\text { unidades de Estratégia de Saúde da Família cobrem do } \\
\text { total da população. }\end{array}$ & Datasus & $(-)$ \\
\hline
\end{tabular}

Fonte: Elaborado pelos autores.

É importante destacar que, em relação à variável NMAT, a identificação das matrículas foi realizada pelo local de nascimento dos indivíduos com o intuito de evitar que o fluxo migratório de alunos do interior para grandes centros inflasse o nível de escolaridade de regióes metropolitanas. A escolha por essa variável se deve pelo fato de não existir fonte municipal de nível escolaridade ano a ano. Assim, espera-se que esta variável seja capaz de refletir, de alguma forma, o efeito da educação no conhecimento e hábitos saudáveis dos indivíduos.

O período de tempo escolhido para realizar a análise foi 2000-2017 em virtude de esse ser o momento em que as bases de dados utilizadas serem compatíveis com informações completas para os municípios. Além disso, é necessário ressaltar que as análises contemplaram 91 dos 92 municípios fluminenses. Isso ocorreu devido a cidade de Mesquita ter surgido em meados de 2001 e, portanto, não haveria informações anteriores a esse período. 
A variável de maior interesse, que mede o impacto das UPAs sobre ICSAP, foi construída com base na metodologia do modelo de diferenças em diferenças, buscando capturar o efeito dessa política nos municípios contemplados com as unidades. O detalhamento dessa variável está na seção a seguir.

\subsection{O modelo empírico}

Para analisar o impacto das UPAs sobre as ICSAP foi escolhido o método de diferenças em diferenças (DD) para dados em painel. Essa técnica permite isolar o efeito de interesse por meio da comparação entre um grupo que recebeu o tratamento e outro grupo que não recebeu. No contexto deste estudo, o município $i$ pertencerá ao grupo de tratados $(j=T)$ ou ao grupo de controle $(j=\mathrm{C})$ na medida em que as instalações das UPAs são realizadas. Convém destacar também que o uso do DD requer que existam observações para os grupos de controle e tratado antes e depois da ocorrência do tratamento. Assim, dado que as inaugurações das UPAs se iniciaram em 2007, o período $t$ de tratamento será denotado por $t=1$ para os anos 2007 em diante e $t=0$ para períodos anteriores. A equação (1) descreve o modelo DD para o estudo em questão:

$$
\operatorname{ICSAP}_{i t}=\alpha+\beta_{1} T_{i}+\beta_{2} D_{t}+\beta_{3} T_{i} * D_{t}+\delta x_{i t}+u_{i t}
$$

onde $T_{i}$ representa uma variável binária que assume valor 1 se o município pertencer ao grupo de tratados e 0 caso contrário; $D_{t}$ é uma dummy que assume 1 se o período for igual ou posterior ao início da política de implementação das UPAs, caso contrário seu valor é nulo; Já $T_{i} * D_{t}$ busca mensurar a interação dessas variáveis, ou seja, evidencia o município $i$ tratado no período $t$. O termo $\delta$ representa o coeficiente estimado para cada variável de controle da matriz $X_{i t}$ e $u_{i t}$ é o termo de erro do modelo.

Com base na equação (1) é possível decompor os resultados dos grupos envolvidos em: grupo de controle antes do programa e depois e grupo de tratados antes e depois do programa. Para o grupo de controle, o valor esperado das ICSAP antes da inauguração das UPAs será dado pela equação (2):

$$
E[I C S A P \mid X, T=0, D=0]=\delta_{i} \hat{x}_{i}
$$


Após o tratamento, a estimativa para o grupo de controle será:

$$
E[I C S A P \mid X, T=0, D=1]=\delta_{j} \hat{x}_{j}+\hat{\beta}_{2}
$$

O grupo de tratamento, por sua vez, tem sua modelagem antes do tratamento conforme a equação (4):

$$
E[\operatorname{ICSAP} \mid X, T=1, D=0]=\delta_{i} \hat{x}_{i}+\hat{\beta}_{2}
$$

Com as inaugurações das UPAs, espera-se que o grupo de tratados tenha o seguinte impacto sobre as ICSAP:

$$
E[I C S A P \mid X, T=1, D=1]=\delta_{j} \hat{x}_{j}+\hat{\beta}_{2}+\hat{\beta}_{3}
$$

A lógica por trás do estimador DD consiste em fazer a diferença no grupo de controle (equações 2 e 3 ) e no grupo de tratamento (equações 4 e 5). Após essa diferença, calcula-se a diferença dessas diferenças, resultando em $\hat{\beta}_{3}$. Assim, esse parâmetro mostra o impacto do programa de implementação das UPAs nas taxas ICSAP.

Em relação à matriz de variáveis de controle, convém destacar a inclusão da variável ESF. Essa variável foi incluída no modelo para evitar que o coeficiente $\hat{\beta}_{3}$ de interesse fosse superestimado. Segundo as diretrizes para implantação das UPAs, estas unidades devem se situar, preferencialmente, em locais com demanda por saúde elevada. Por conseguinte, também é sabido que o programa ESF obteve uma expansão considerável ao longo dos anos 2000, permitindo que houvessem avanços em saúde nos municípios. Dessa forma, ao introduzir esta variável busca-se isolar a influência de qualquer efeito decorrente do progresso em saúde devido as ESF e identificar o impacto das UPAs nas ICSAP.

Nesse sentido, o impacto das UPAs sobre as ICSAP é fundamentado na ideia de que, condicionado aos controles do modelo, não existirão outras fontes de ICSAP que se correlacionem com as UPAs e, portanto, seu efeito será identificado considerando a suposição de tendência comuns entre os grupos de tratamento e controle. 


\section{Análise dos Resultados e Discussão}

Inicialmente, foi realizada uma análise descritiva das variáveis do estudo para melhor conhecimento de seu comportamento. A Tabela 1 apresenta o comportamento das variáveis antes e depois do período de inauguração das UPAs nos grupos de controle e tratamento.

Tabela 1 - Comparação de Médias das variáveis para os municípios antes e depois das UPAs

\begin{tabular}{ccccccc}
\hline & \multicolumn{3}{c}{ Período pré UPA (2000-2006) } & \multicolumn{3}{c}{ Período pós UPA (2007-2017) } \\
\hline Variável & Controle & Tratamento & $\begin{array}{c}\text { Diferença de } \\
\text { médias }\end{array}$ & Controle & Tratamento & $\begin{array}{c}\text { Diferença de } \\
\text { médias }\end{array}$ \\
\hline \multirow{2}{*}{ Água (\%) } & 79,76 & 76,37 & 3,39 & 83,12 & 85,85 & $-2,73$ \\
& $(26,46)$ & $(26,59)$ & {$[1,75]$} & $(25,29)$ & $(21,75)$ & {$[1,44]$} \\
Esgoto (\%) & 40,55 & 41,71 & $-1,16$ & 58,53 & 56,73 & 1,79 \\
& $(34,97)$ & $(37,10)$ & {$[12,88]$} & $(26,15)$ & $(28,71)$ & {$[2,20]$} \\
Pib (Mil R\$) & 8,20 & 10,26 & $-2,06$ & 10,44 & 12,17 & $-1,73$ \\
& $(13,49)$ & $(10,15)$ & {$[1,07]$} & $(18,52)$ & $(13,85)$ & {$[1,04]$} \\
ESF (\%) & 46,45 & 30,09 & $16,36 * * *$ & 85,89 & 70,17 & $15,71^{* * *}$ \\
& $(34,83)$ & $(26,07)$ & {$[2,52]$} & $(23,04)$ & $(26,69)$ & {$[1,58]$} \\
NMAT (mil/hab) & 18,76 & 19,71 & $-0,95$ & 22,17 & 24,68 & $-2,51$ \\
& $(13,17)$ & $(14,23)$ & {$[15,86]$} & $(14,37)$ & $(22,64)$ & {$[2,85]$} \\
ICSAP (100mil/hab) & 2350,12 & 2180,10 & 170,02 & 2020,38 & 1010,30 & $1010,08^{* * *}$ \\
& $(1103,74)$ & $(1214,78)$ & {$[98,32]$} & $(1132,07)$ & $(672,14)$ & {$[16,37]$} \\
\hline N & 400 & 328 & & 500 & 410 & \\
\hline
\end{tabular}

Fonte: Dados da pesquisa (2020).

Nota: Desvio padrão entre parênteses e erro-padrão entre colchetes.

Obs: ${ }^{* * *} 1 \%$ de significância.

Observando a tabela acima é possível constatar que os municípios no grupo de tratamento e controle possuem médias semelhantes dentre quase todas as variáveis apresentadas, excetuando-se ESF que apresentou diferenças estatisticamente significativas em suas médias ao longo do tempo. Nas ICSAP já era esperada a diferença tendo em vista que a média apresentada para o período 2007-2017 já contabiliza a existência das UPAs. Em geral, foi observado que todas as variáveis exibiram tendência comum de crescimento em ambos os grupos. A exceção foi a variável ICSAP, denotando que esta já apresentava uma tendência de queda aproximada de 3,05\% anualmente. Esse fato pode ser explicado pelo avanço dos serviços de água e esgoto que, com o Programa de Aceleração do Crescimento (PAC) e a Lei 11.445/2007 (marco regulatório do saneamento no Brasil), possibili- 
tou a expansão de investimentos neste setor, aumentando o percentual de pessoas atendidas.

Além disso, ocorreu a expansão do Programa de Estratégia de Saúde da Família (ESF) que, apesar de ter sofrido algumas variações negativas (entre 2000-2001, 2009-2010 e entre 2016-2017), seu crescimento médio anual foi de $6 \%$. Esse resultado evidencia o esforço do governo em ampliar os atendimentos na atenção primária de saúde. Entretanto, deve-se ressaltar que esse movimento de ampliação visa universalizar o acesso saúde e que para se alcançar a cobertura universal é necessário que se avance em outros programas do governo, que ofereçam outros serviços de saúde, como por exemplo, as UPAs.

Com relação às ICSAP, no período anterior à existência das UPAs foi constatado que o grupo de controle possuía cerca de $7,5 \%$ a mais de internações se comparado ao grupo tratado. Por outro lado, quando se analisa o período pós-implantação das UPAs, apesar da tendência se repetir (mais internações no grupo de controle), as ICSAP se reduziram com mais intensidade nos municípios que foram tratados, fazendo com que o grupo de controle obtivesse duas vezes mais internações. Isso mostra que as UPAs podem ter contribuído para descongestionar as emergências hospitalares com suas consultas ambulatoriais emergenciais, dado que as demais variáveis obtiveram comportamento semelhante em ambos os grupos pesquisados.

A seguir, na Tabela 2, são apresentados os resultados da sequência de regressões para modelo DD com a inclusão das variáveis discutidas na metodologia. Ressalta-se que durante o procedimento de análise exploratória dos dados foi identificado um pico repentino no crescimento das ICSAP para o ano de 2013. Esse aumento inesperado nos resultados pode ser explicado, em grande parte, devido às fortes chuvas que castigaram a região metropolitana fluminense onde famílias ficaram desalojadas em virtude dos alagamentos e deslizamentos. É sabido que quando ocorre esse tipo de fenômeno, muitos indivíduos (principalmente crianças e pessoas com saúde fragilizada) acabam sendo internados por entrar em contato com a água contaminada. Assim, foi adicionada uma dummy (chuva_2013) no modelo para os municípios ${ }^{3}$ que foram afetados por esse choque exógeno. Além disso, também foi adicionada a estrutura da estimação do modelo proposto

3 Belford Roxo, Duque de Caxias, Guapimirim, Itaboraí, Itaguaí, Japerí, Magé, Maricá, Mesquita, Nilópolis, Niterói, Nova Iguaçu, Paracambi, Petrópolis, Queimados, Seropédica, São Gonçalo, São João de Merití, Tanguá, Cachoeiras de Macacu e Rio Bonito. 
pela equação 1 efeitos fixos de tempo e município. Considerar a adição desses efeitos é importante garantir a comparabilidade do método Diff in Diff além de capturar possíveis efeitos de heterogeneidade individual. Os resultados estão expostos na Tabela 2.

Tabela 2 - Análise do impacto das UPAs nas ICSAP controlando por outras variáveis

\begin{tabular}{|c|c|c|c|c|c|}
\hline Variável & Modelo I & Modelo II & Modelo III & Modelo IV & Modelo V \\
\hline$\hat{\beta}_{3}$ & $\begin{array}{c}-22,45^{\star \star \star} \\
(-4,34)\end{array}$ & $\begin{array}{c}-16,77^{\star \star \star} \\
(3,77)\end{array}$ & $\begin{array}{c}-17,87^{\star \star \star} \\
(3,85)\end{array}$ & $\begin{array}{c}-19,71^{\star \star \star} \\
(3,93)\end{array}$ & $\begin{array}{c}-21,44^{\star \star \star} \\
(4,17)\end{array}$ \\
\hline$\hat{\beta}_{2}$ & $\begin{array}{c}-63,45^{\star \star *} \\
(4,41)\end{array}$ & $\begin{array}{c}-62,21^{\star \star \star} \\
(6,01)\end{array}$ & $\begin{array}{c}-62,00^{\star \star *} \\
(6,65)\end{array}$ & $\begin{array}{c}-60,06^{\star \star \star} \\
(7,09)\end{array}$ & $\begin{array}{c}-64,52^{\star \star \star} \\
(7,65)\end{array}$ \\
\hline$\hat{\beta}_{1}$ & $\begin{array}{c}-944,90^{\star * *} \\
(46,27)\end{array}$ & $\begin{array}{c}-1438,40^{* * *} \\
(86,96)\end{array}$ & $\begin{array}{c}-1535,90^{* * *} \\
(88,01)\end{array}$ & $\begin{array}{c}-1554,40^{* * *} \\
(103,14)\end{array}$ & $\begin{array}{c}-1492,10^{* * *} \\
(139,91)\end{array}$ \\
\hline \multicolumn{6}{|c|}{ Controles } \\
\hline PIB & & $\begin{array}{l}-4,80 \\
(2,58)\end{array}$ & $\begin{array}{c}-8,07^{\star \star} \\
(2,65)\end{array}$ & $\begin{array}{c}-6,54^{\star \star} \\
(2,56)\end{array}$ & $\begin{array}{r}-4,56^{*} \\
(2,23)\end{array}$ \\
\hline ESF & & & $\begin{array}{l}-0,50 \\
(0,86)\end{array}$ & $\begin{array}{l}-0,48 \\
(0,86)\end{array}$ & $\begin{array}{l}-0,50 \\
(0,96)\end{array}$ \\
\hline NMAT & & & & $\begin{array}{l}2,68^{*} \\
(1,30)\end{array}$ & $\begin{array}{l}3,60^{* *} \\
(1,33)\end{array}$ \\
\hline $\mathrm{Ag}$ & & & & & $\begin{array}{l}-1,28 \\
(1,17)\end{array}$ \\
\hline Chuva_2013 & & & & & $\begin{array}{c}57,45 \\
(56,26)\end{array}$ \\
\hline Constante & $\begin{array}{c}129664,80^{* * *} \\
(8862,93)\end{array}$ & $\begin{array}{c}127115,30^{* * *} \\
(8815,20)\end{array}$ & $\begin{array}{c}159815,00^{* * *} \\
(10976,30)\end{array}$ & $\begin{array}{c}122719,60^{* \star *} \\
(14227,37)\end{array}$ & $\begin{array}{l}131708^{\star * \star} \\
(15366,60)\end{array}$ \\
\hline $\begin{array}{l}\text { Efeitos fixos de } \\
\text { tempo e município }\end{array}$ & Não & Sim & Sim & Sim & Sim \\
\hline Observações & 1638 & 1608 & 1602 & 1522 & 1353 \\
\hline Tratamento & 697 & 697 & 697 & 697 & 697 \\
\hline
\end{tabular}

Fonte: Dados da pesquisa (2020).

Obs: Erros-padrão robustos nos parênteses.

Nota: ${ }^{* *} 0,1 \%$ de significância; ${ }^{* *} 1 \%$ de significância; * $5 \%$ de significância.

Os resultados ${ }^{4}$ da Tabela 2 indicaram que o coeficiente $\hat{\beta}_{3}$ (mensura o efeito das UPAs na taxa de ICSAP) apresentou forte significância estatística em todos os modelos econométricos e com o sinal esperado. Isso sugere que as UPAs conseguem cumprir com seu papel como unidade secundária

4 A variável esgoto foi excluída da análise de regressão por esta reduzir a base de dados pela metade. É importante mencionar também que o SNIS alterou a forma de cálculo desta variável o que poderia ocasionar distorções nas análises. 
de atendimento em saúde, podendo evitar hospitalizações desnecessárias se tratadas em consultas emergenciais. Todavia, deve-se considerar a possibilidade de sobrecarga dessa estrutura de saúde uma vez que a variável ESF, outra componente potencial em reduzir ICSAP, não obteve significância neste estudo.

O fato das UPAs atenderem urgências e emergências garante, em muitas ocasiões, a realização de exames rapidamente, contribuindo para um diagnóstico mais rápido e preciso da enfermidade. Por outro lado, em unidades ESF, a realização de exames laboratoriais fica condicionada a autorização pelas secretarias municipais de saúde, ocasionando uma fila de espera que pode ser de dias ou meses. Logo, essa característica parece influenciar na resolutividade das consultas realizadas em cada estrutura de saúde, tornando as UPAs um instrumento de saúde pública fundamental no sistema de saúde brasileiro.

Os resultados encontrados para os coeficientes que medem o efeito do tratamento nos grupos dos tratados $\left(\hat{\beta}_{1}\right)$ e o efeito do tratamento no tempo $\left(\hat{\beta}_{2}\right)$ foram significativos e mantiveram seus sinais esperados ao longo dos modelos. Apesar de isoladamente não capturarem o impacto da implementação das UPAs, esses achados apontam que, após a inauguração das UPAs, as ICSAP sofreram queda dentro dos municípios tratados e ao longo do tempo na amostra pesquisada.

Com relação aos controles, os resultados da Tabela 2 mostraram que o percentual da população urbana atendida com água encanada (Ag) e a dummy para capturar o efeito das chuvas em 2013 sobre a taxa de ICSAP não foram significativos no modelo completo (modelo $\mathrm{V}$ ). Isso revela que, mesmo havendo um pico nos casos de ICSAP para o ano 2013, as chuvas não foram determinantes para essa ocorrência. Além disso, vale ressaltar que o fato de um maior atendimento da população urbana com água encanada não apresentar significância estatística não significa que essa variável seja irrelevante. Pelo contrário, como o abastecimento urbano se encontra acima dos $80 \%$ em ambos os grupos, isso sugere que a desigualdade no acesso possa ser mais relevante do que a expansão propriamente dita. Outras variáveis de controle (PIB e NMAT) exibiram significância estatística. O PIB per capita apresentou o sinal esperado, evidenciando que municípios com mais renda per capita têm menores taxas por ICSAP conforme autores como Wagstaff (1993) e Castro, Travassos e Carvalho (2005) destacavam. 
Entretanto, a taxa de matrícula por mil habitantes dos matriculados e concluintes no ensino superior (NMAT) obteve sinal diferente ao que era esperado. Esse fato pode ser explicado com um contraexemplo ao que foi mencionado por Grossman (1972). Para o autor, maior escolaridade implicaria em um melhor conhecimento sobre suas condições de saúde, o que levaria o indivíduo a buscar cuidados médicos no momento adequado, evitando internações desnecessárias. Porém, por conhecer melhor seu status de saúde, esses indivíduos podem retardar sua demanda por cuidados até o momento em que considere que sua condição será caracterizada como emergência/urgência e assim buscar um serviço hospitalar. Isso ocorre porque, como Politi (2014) já havia destacado, existe uma enorme desigualdade pró ricos para o acesso de consulta médicas especializadas ou procedimentos de alta complexidade.

Em relação ao Programa de Estratégia de Saúde da Família (ESF), a não significância de seu coeficiente pode estar relacionado com as dificuldades do sistema público de saúde em dar prosseguimento nas demandas atendidas nos postos de saúde. O indivíduo que é atendido nas unidades ESF pode ser encaminhado para consultas especializadas e/ou realização de exames específicos para um melhor diagnóstico, seja qual for a morbidade. Porém, principalmente para a realização de exames, é requerido que exista disponibilidade de recursos que, em muitas ocasiões, não são liberados no tempo necessário. Assim, apenas a ampliação das ESF pode não ser suficiente para que essas hospitalizações se reduzam, pois, conforme já havia sido identificado por Politi (2014), é necessário que novas estratégias sejam desenvolvidas para mitigar as desigualdades no acesso de procedimentos mais especializados (consulta/exames) e, nesse sentido, as UPAs podem surgir como uma alternativa.

Com o intuito de verificar a robustez dos resultados encontrados na Tabela 2, foi realizada uma sequência de testes placebo para verificar se a especificação do modelo mais completo (modelo V da Tabela 2) estava adequada. Além das ICSAP, as internações por neoplasias (câncer) e para a realização de partos estão entre as principais causas de hospitalizações. Assim, o exercício de robustez considerou um modelo com a variável dependente sendo a taxa de internação por neoplasias por 100 mil habitantes e outro com a taxa de internação para partos por 100 mil habitantes. A Tabela 3 apresenta os resultados das regressões. 
Tabela 3 - Comparação entre resultados do modelo $\mathrm{V}$ e outras internações (teste de robustez)

\begin{tabular}{cccc}
\hline Variável & Modelo V & Modelo VI & Modelo VII \\
\hline$\hat{\beta}_{3}$ & $-21,44^{* * *}$ & $-0,0011$ & $-0,00070$ \\
& $(4,17)$ & $(0,0014)$ & $(0,0011)$ \\
$\hat{\beta}_{2}$ & $-64,52^{* * *}$ & 0,000335 & $-0,000684^{*}$ \\
& $(7,65)$ & $(0,00039)$ & $(0,00017)$ \\
$\hat{\beta}_{1}$ & $-1492,10^{* * *}$ & $0,00275^{\star * *}$ & $0,0163^{* * *}$ \\
& $(139,91)$ & $(0,00049)$ & $(0,0034)$ \\
\hline \multirow{2}{*}{ PIB } & & & $0,000689^{*}$ \\
& $-4,56^{*}$ & 0,0000075 & $(0,00032)$ \\
ESF & $(2,23)$ & $(0,0000063)$ & $-0,000729^{* * *}$ \\
& $-0,50$ & $-0,0000050$ & $(0,000024)$ \\
NMAT & $(0,96)$ & $(0,000033)$ & 0,00015 \\
& $3,60^{* *}$ & 0,00068 & $(0,00011)$ \\
Ag & $(1,33)$ & $(0,000076)$ & $-0,000098$ \\
& $-1,28$ & $-0,0000714^{*}$ & $(0,00049)$ \\
Chuva_2013 & $(1,17)$ & $(0,000033)$ & 0,00186 \\
& 57,45 & $-0,00283$ & $(0,0014)$ \\
Constante & $(56,26)$ & $(0,0016)$ & $-1,379^{* * *}$ \\
& $131708^{* * *}$ & $-0,06635$ & $(0,36)$ \\
\hline municípios & $(15366,60)$ & $(0,075)$ & Sim \\
\hline Observações & Sim & Sim & 1353 \\
\hline Tratamento & 1353 & 1353 & 697 \\
\hline
\end{tabular}

Fonte: Dados da pesquisa (2020).

Obs: Erros-padrão robustos nos parênteses.

Nota: ${ }^{* * *} 0,1 \%$ de significância; ${ }^{* *} 1 \%$ de significância; * $5 \%$ de significância.

Além do modelo V (já apresentado na tabela 2), a tabela 3 apresenta o impacto das UPAs nas internações por neoplasias (modelo VI) e partos (modelo VII) como exercício de robustez. Os resultados evidenciaram que não há significância estatística da implementação das UPAs sobre as internações por neoplasias e gravidez, confirmando a adequação do modelo de diferenças em diferenças aplicado para avaliar o impacto da UPAs. 


\section{Considerações Finais}

Objetivou-se com este estudo avaliar o impacto das UPAs na demanda por internações sensíveis à atenção primária nos municípios do estado do Rio de Janeiro, utilizando um modelo de diferenças em diferenças. Inicialmente, ficou constatado que a estratégia empírica para estimar o modelo DD se mostrou adequada, pois os testes placebos que foram realizados confirmaram a aderência do DD em capturar o efeito da implementação das UPAs com robustez.

Os resultados indicam que as UPAs têm um efeito negativo e significativo nas taxas ICSAP, caracterizando essas unidades de saúde como um instrumento relevante na redução da superlotação em emergências hospitalares. Em média, os municípios com UPA instalada reduziram as ICSAP em cerca de $21\left(\hat{\beta}_{3}\right)$ unidades por 100 mil habitantes ao longo do período em que houve o tratamento. Considerando esse resultado, juntamente com o custo médio dessas internações em $\mathrm{R} \$ 1.017,77$ e a totalidade de municípios desse estudo, o produto entre eles poderia resultar em uma economia aos cofres públicos de aproximadamente $\mathrm{R} \$ 920$ mil reais ao final de um ano.

Entretanto, enfatizar esse resultado para priorizar uma possível política pública de expansão dessas unidades pode não ser o mais adequado. Sabendo que a maioria da demanda das UPAs é proveniente de casos da atenção primária, é necessário compreender quais problemas são enfrentados na porta de entrada do SUS. Dessa forma, uma estratégia mais eficiente pode ser traçada para evitar que as UPAs cumpram o papel de hospitais e funcionem, de fato, como Unidades de Pronto Atendimento.

Embora o estudo tenha alcançado de forma satisfatória o objetivo, ainda é necessário fazer algumas ressalvas quanto as suas limitações. O fato de algumas variáveis de controle não apresentarem os sinais esperados pode dificultar o delineamento de políticas públicas que integrem essas áreas para um esforço de redução das ICSAP. Todavia, estudos que abarquem outros estados brasileiros em diferentes regiões podem contribuir para que os efeitos desejados nessas variáveis sejam identificados. Além disso, pesquisas que desmembrem as ICSAP de acordo com sua especialidade podem contribuir de forma relevante para uma melhor identificação das demandas atendidas não somente nas UPAs, mas também nas ESF. Com maior conhecimento do perfil de cada estrutura de saúde é possível atingir uma maior integração entre a atenção primária, secundária e hospitalar, garantindo o melhoramento dos serviços públicos de saúde. 


\section{Referências}

Boing, A. F, R. B. Vicenzi, F. Magajewski, A. C. Boing, R. O. Pires, K. G. Peres, S. R. Linder, e M. A. Peres. 2012. "Redução das internações por condições sensíveis à atenção primária no Brasil entre 1998-2009". Rev Saúde Pública 46, no. 2: 359-366.

Brito, R. J. A., M. V. Andrade, eI. H. O. Perpétuo. 2006. "Demanda por serviços de saúde”. In: Gasto e consumo das familias brasileiras contemporâneas. Brasília: IPEA.

Castro, M. S. M, C. Travassos, e M. S. Carvalho. 2005. "Efeito da oferta de serviços de saúde no uso de internações hospitalares no Brasil”. Rev Saúde Pública 39, no. 2: 277-284.

Figueiredo, N. D, L. M. Maximo, M. E. Queiroz, K. M.Oliveira, F. Z. Kornalewski, L. S. Ribeiro, e R. Araujo. 2012. "Perfil de atendimento de uma UPA e seu impacto na saúde pública". Artigo apresentado no $10^{\circ}$ Congresso Internacional da Rede Unida, Rio de Janeiro, maio de 2012.

Fiocruz. 2015. "Que sistema de saúde nós queremos ter?" Disponível em: $<\underline{\text { http://www6.ensp.fiocruz.br/ }}$ visa/?q=node/6562 $>$. Acesso em 17 abr. 2020.

Galama, T, e A. Kapteyn. 2011. "Grossman's missing health threshold". Journal of Health Economics 30: 1044-1056.

Grossman, M. 1972. "The Demand for Health: A theorical and empirical investigation”. New York: National Bureau of Economic Research. 1972.

Iunes, R. F. 1995. "Demanda e demanda por saúde. Economia da Saúde: Conceito e contribuição para a gestão da saúde”. Brasília: IPEA.

Konder, M. 2013. "Atenção às urgências: a integração das Unidades de Pronto Atendimento 24h (UPA 24h) com a rede assistencial do município do Rio de Janeiro". Dissertação de mestrado em Saúde Pública, Escola Nacional de Saúde Pública Sérgio Arouca, ENSP.

Konder, M. T, e G. O'dwyer. 2016. "A integração das unidades de pronto atendimento (UPA) com a rede assistencial no município do Rio de Janeiro". Interface: Communication, Health, Education 20, no. 59: 879-892.

Lima, A. C, L. Y. Nichiata, e D. Bonfim. 2019. "Perfil dos atendimentos por condições sensíveis à atenção primária à saúde em uma Unidade de Pronto Atendimento". Rev Esc Enfermagem USP 53, no. 1: 1-7.

Ministério da Saúde. 2011. Portaria $\mathrm{n}^{\circ}$ 1.601, de 7 de julho de 2011. "Estabelece diretrizes para a implantação do componente Unidades de Pronto Atendimento (UPA24h) e o conjunto de serviços de urgência 24 horas da Rede de Atenção às Urgências, em conformidade com a Política Nacional de Atenção às Urgências". Diário Oficial da União: 70-70.

Ministério da Saúde. 2014. Esclarecimento sobre leitos no SUS (2014). Disponível em: $<$ http://www.blog.saude. gov.br/e42el5>. Acesso em: 15 abr. 2020.

Moura, B. L, R. C. Cunha, R.Aquinho, M. G. Medina, E. L. Mota, J. Macinko, e I. Dourado. 2010. "Principais causas de internação por condições sensíveis à atenção primária no Brasil: Uma análise por faixa etária e região". Rev. Bras. Saúde Matern. Infant. 10, no. 1: S83-S91.

O'dwyer, G. O, S. P. Oliveira, e M. H. Seta. 2009. "Avaliação dos serviços hospitalares de emergência do programa QualiSUS”. Ciência e Saúde Coletiva 14, no. 5: 1881-1889.

O'dwyer, G, M. T. Konder, L. P. Reciputti, e M. G. Lopes. 2017. "O processo de implantação das unidades de pronto atendimento no Brasil”. Revista de Saúde Pública 51: 1-12.

Pinto, R. S, T. Stocker, e T. M. Lima. 2019. "O papel das unidades de pronto atendimento: análise do desempenho da primeira UPA do município de Pelotas-RS". Revista de Gestão em Sistemas de Saúde 8, no. 2: 127-136.

Politi, R. 2014. "Desigualdade na utilização de serviços de saúde entre adultos: uma análise dos fatores de concentração da demanda". Economia Aplicada 18, no. 1: 117-137. 
Santos, L. P, A. L. Castro, V. G. Dutra, e R. M. Guimarães. 2018. "Internações por condições sensíveis à atenção primária à saúde, 2008-2015: uma análise do impacto da expansão da ESF na cidade do Rio de Janeiro". Cad. Saúde Colet 26, no. 2.

Secretaria Estadual de Saúde do Estado do Rio de Janeiro. 2010. "Projeto básico de gestão e administração de Unidades de Pronto Atendimento, 2010". Disponível em: $<$ https://www.saude.rj.gov.br/comum/code/MostrarArquivo.php? $\mathrm{C}=\mathrm{NTA} 1 \mathrm{NA} \% 2 \mathrm{C} \% 2 \mathrm{C}>$. Acesso em 12 abr. 2020.

Silva, G. S, I. Samico, L. S. Dubeux, e E. Felisberto. 2012. "Redes de atenção às urgências e emergências: Pré-avaliação das unidades de pronto atendimento (UPAs) em uma região metropolitana do Brasil". Revista Brasileira de Saúde Materno Infantil 12, no. 4: 445-458.

Silva, K, e S. J. C. Gonçalves. 2019. “Avaliação ecológica de internações por condições sensíveis à atenção primária no município de Miguel Pereira-RJ”. Revista Pró-univerSUS 10, no. 1: 119-124.

Wagstaff, A. 1993. "The demand for health: an empirical reformulation Grossman model". Health Economics 5: 193-233.

Zarlotti, C, E. Scudese, G. W. Senna, T. Tonini, T. S. Lopes, e C. L. Pestana . 2017. "Internações por condições sensíveis à atenção primária após a implantação da estratégia de saúde da família no município de Petrópolis/ RJ". Rev Fund Care online 9, no. 3: 811-817. 